\title{
A Note on Differencing the Viscous Dissipation Terms for a Newtonian Fluid
}

\author{
L. D. Cloutman
}

June 1, 2001

U.S. Department of Energy

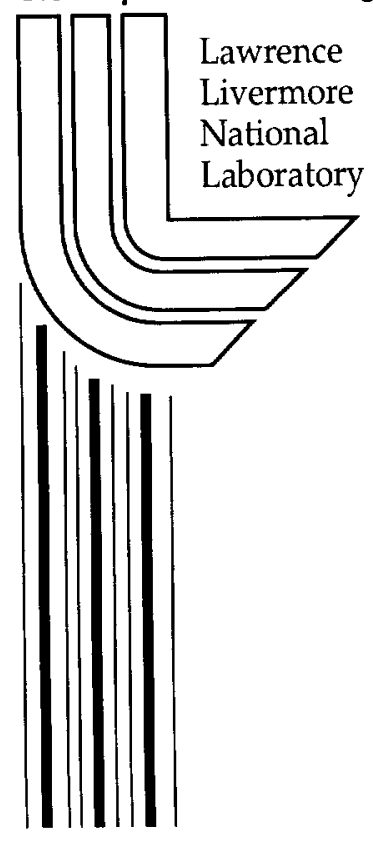




\section{DISCLAIMER}

This document was prepared as an account of work sponsored by an agency of the United States Government. Neither the United States Government nor the University of California nor any of their employees, makes any warranty, express or implied, or assumes any legal liability or responsibility for the accuracy, completeness, or usefulness of any information, apparatus, product, or process disclosed, or represents that its use would not infringe privately owned rights. Reference herein to any specific commercial product, process, or service by trade name, trademark, manufacturer, or otherwise, does not necessarily constitute or imply its endorsement, recommendation, or favoring by the United States Government or the University of California. The views and opinions of authors expressed herein do not necessarily state or reflect those of the United States Government or the University of California, and shall not be used for advertising or product endorsement purposes.

This work was performed under the auspices of the U. S. Department of Energy by the University of California, Lawrence Livermore National Laboratory under Contract No. W-7405-Eng-48.

This report has been reproduced directly from the best available copy.

Available electronically dttp://www.doc.gov/bridge

Available for a processing fee to U.S. Department of Energy

And its contractors in paper from

U.S. Department of Energy

Office of Scientific and Technical Information

P.O. Box 62

Oak Ridge, TN 37831-0062

Telephone: (865) 576-8401

Facsimile: (865) 576-5728

E-mail:reports@adonis.osti.gov

Available for the sale to the public from

U.S. Department of Commerce

National Technical Information Service 5285 Port Royal Road Springfield, VA 22161

Telephone: (800) 553-6847

Facsimile: (703) 605-6900

E-mail:orders@ntis.fedworld.gov

Online ordering: http://www.ntis.gov/ordering.htm

OR

Lawrence Livermore National Laboratory

Technical Information Department's Digital Library

http://www.llnl.gov/tid/Library.html 
Lawrence Livermore National Laboratory Report

UCRL-ID-??????

June 2001

\title{
A NOTE ON DIFFERENCING THE VISCOUS DISSIPATION TERMS FOR A NEWTONIAN FLUID
}

\author{
Lawrence D. Cloutman
}

Lawrence Livermore National Laboratory

\begin{abstract}
We compare two finite difference approximations for the viscous dissipation terms in the energy equation. We focus on the strain produced by the every-other-zone (e.g., hour-glass and herringbone) modes in the velocity field. Care must be exercised to produce a viscous dissipation rate consistent with the viscous forces. The examples given are for a rectangular staggered grid, but similar considerations apply to other types of grids. Also, these considerations apply to certain algebraic eddy viscosity models and to the shear creation terms in turbulence transport models.
\end{abstract}




\section{Introduction}

Most of the research effort on finite differencing the transient equations of fluid dynamics has been put into the convection terms and time marching schemes. As important as that effort has been, we cannot neglect the rest of the terms in the equations. In this report, we focus attention on the finite difference approximation used for the viscous dissipation term in the energy equation. As a concrete example, we use the COYOTE computational fluid dynamics (CFD) code [1] to show how the choice of difference methods can influence accuracy in subtle but significant ways. Terms with the same mathematical form as the viscous dissipation appear in various turbulence models, and this discussion applies to them as well.

In Section 2, we present the basic governing equations. Section 3 summarizes some relevant equations from standard turbulence models. Section 4 expands these equations into components in Cartesian and cylindrical coordinates, a necessary step in creating the finite difference equations. Section 5 discusses some considerations on how to difference the viscous terms, and Section 6 gives numerical examples. We conclude with the summary in Section 7.

\section{Governing Equations}

We consider the single-velocity multicomponent Navier-Stokes equations. Mass conservation is expressed by the continuity equation for each species $\alpha$ :

$$
\frac{\partial \rho_{\alpha}}{\partial t}+\nabla \cdot\left(\rho_{\alpha} \mathbf{u}\right)=-\nabla \cdot \mathbf{J}_{\alpha}+R_{\alpha}
$$

where $\rho_{\alpha}$ is the density of species $\alpha, t$ is time, $\mathbf{u}$ is the velocity, and $R_{\alpha}$ is the rate at which species $\alpha$ is created by chemical reactions. Calculation of the exact diffusional mass fluxes is a complicated proposition (for example, Bird, Stewart, and Lightfoot [2] and Chapman and Cowling [3]), but it will suffice for our present purpose to simply consider Fick's law,

$$
\mathbf{J}_{\alpha}=-\rho D \nabla\left(\rho_{\alpha} / \rho\right)
$$

where $\rho$ is the total density, and $D$ is the species diffusivity, which we assume is independent of species. Equation 1 may be summed over species to obtain the total continuity equation

$$
\frac{\partial \rho}{\partial t}+\nabla \cdot(\rho \mathbf{u})=0
$$

The momentum equation is

$$
\frac{\partial(\rho \mathbf{u})}{\partial t}+\nabla \cdot(\rho \mathbf{u u})=\rho \mathbf{g}-\nabla P+\nabla \cdot \mathrm{T},
$$


where $\mathbf{g}$ is the gravitational acceleration, $P$ is the pressure, and $\mathrm{T}$ is the stress tensor

$$
\begin{gathered}
\mathrm{T}=\mu\left[\nabla \mathbf{u}+(\nabla \mathbf{u})^{T}\right]+\mu_{1} \nabla \cdot \mathbf{u} \mathbf{U} \\
=\mu\left[\nabla \mathbf{u}+(\nabla \mathbf{u})^{T}-\frac{2}{3} \nabla \cdot \mathbf{u} \mathbf{U}\right]+\mu_{\mathrm{b}} \nabla \cdot \mathbf{u} \mathbf{U} .
\end{gathered}
$$

Here $U$ is the unit tensor, and $\mu$ is the coefficient of viscosity,

$$
\mu_{1}=\mu_{\mathrm{b}}-2 \mu / 3
$$

is the second coefficient of viscosity, and $\mu_{\mathrm{b}}$ is the bulk viscosity.

We express energy conservation in terms of the specific thermal internal energy $I$ :

$$
\frac{\partial(\rho I)}{\partial t}+\nabla \cdot(\rho I \mathbf{u})=-P \nabla \cdot \mathbf{u}+\mathrm{T}: \nabla \mathbf{u}-\nabla \cdot \mathbf{q}+\sum_{\alpha} H_{\alpha} R_{\alpha}
$$

where $\mathbf{q}$ is the diffusional heat flux, and $H_{\alpha}$ is the heat of formation of species $\alpha$. The heat flux is another complicated function that we approximate as the sum of Fourier's law and enthalpy diffusion:

$$
\mathbf{q}=-K \nabla T+\sum_{\alpha} h_{\alpha} \mathbf{J}_{\alpha}
$$

where $K$ is the conductivity, $h_{\alpha}$ is the enthalpy of species $\alpha$, and $T$ is the temperature. We can also develop models based on the enthalpy $h=I+P / \rho$ and the temperature. These alternative energy equations have the same viscous dissipation term [2].

In what follows it will be convenient to introduce the rate of strain tensor, defined by

$$
\mathrm{E} \equiv 0.5\left[\nabla \mathbf{u}+(\nabla \mathbf{u})^{\mathrm{T}}\right]
$$

Note that

$$
\mathrm{E}: \mathrm{U}=\nabla \mathbf{u}: \mathrm{U}=(\nabla \mathbf{u})^{\mathrm{T}}: \mathrm{U}=\nabla \cdot \mathbf{u}
$$

\section{Turbulence Models}

The $\mathrm{T}: \nabla \mathbf{u}$ term also appears in many different turbulence models. We shall consider two examples: an algebraic large eddy simulation (LES) model and a generic transport equation for the turbulence kinetic energy density. In all of these models, we shall assume that the Reynolds stress is modeled by the familiar Boussinesq approximation

$$
\mathrm{R}=\tilde{\rho} \overline{\mathrm{u}^{\prime} \mathbf{u}^{\prime}}=-\mu_{\mathrm{t}}\left[\nabla \overline{\mathbf{u}}+(\nabla \overline{\mathbf{u}})^{\mathrm{T}}-\frac{2}{3} \mathrm{U} \nabla \cdot \overline{\mathbf{u}}\right]+\frac{2}{3} \tilde{\rho} \mathcal{K} \mathrm{U} \equiv-\mathrm{T}_{\mathrm{t}}+\frac{2}{3} \tilde{\rho} \mathcal{K} \mathrm{U}
$$

where $\mathcal{K}$ is the SGS turbulence kinetic energy density, which is half of the trace of $\mathrm{R}$. Note that $T_{t}$ has the same mathematical form as $T$ with $\mu_{b}=0$ and with the molecular viscosity $\mu$ replaced by the eddy viscosity $\mu_{\mathrm{t}}$. 
The simplest model for the eddy viscosity is the algebraic Smagorinsky model [4],

$$
\mu_{t}=C_{\mathrm{s}}^{2} L^{2} \tilde{\rho}\left\{\nabla \overline{\mathbf{u}}:\left[\nabla \overline{\mathbf{u}}+(\nabla \overline{\mathbf{u}})^{\mathrm{T}}\right]\right\}^{1 / 2}=2^{1 / 2} C_{\mathrm{s}}^{2} L^{2} \tilde{\rho}(\mathrm{E}: \mathrm{E})^{1 / 2}
$$

The value of $C_{\mathrm{s}}$ is selected by comparison of calculations and experiments [5]. Both Smagorinsky and Deardorff normalized this expression by selecting $L=(\delta x \delta y \delta z)^{1 / 3}$, where $\delta x$ is the zone size in the $x$-direction, etc.

The primary turbulent correlations are the turbulence kinetic energy density $\mathcal{K}$ and the Reynolds stress tensor R. Transport equations for these may be derived by formal manipulation of the Navier-Stokes equation. The result for $\mathcal{K}$ is

$$
\begin{gathered}
\frac{\partial \tilde{\rho} \mathcal{K}}{\partial t}+\nabla \cdot\left[\tilde{\rho} \mathcal{K} \overline{\mathbf{u}}+0.5 \tilde{\rho} \overline{u_{i}^{\prime} u_{i}^{\prime} \mathbf{u}^{\prime}}\right]+\mathrm{R}: \nabla \overline{\mathbf{u}} \\
=-[\langle\mathbf{u} \cdot \nabla P\rangle-\overline{\mathbf{u}} \cdot \nabla \tilde{P}]+[\langle\mathbf{u} \cdot(\nabla \cdot \mathrm{T})\rangle-\overline{\mathbf{u}} \cdot(\nabla \cdot\langle\mathrm{T}\rangle)] .
\end{gathered}
$$

One closure for this equation is $[6,7]$

$$
\begin{aligned}
\frac{\partial \tilde{\rho} \mathcal{K}}{\partial t}+\nabla \cdot(\tilde{\rho} \mathcal{K} \overline{\mathbf{u}})=\nabla & \cdot\left[\left(\mu+\mu_{\mathrm{t}}\right) \nabla \mathcal{K}\right]-\frac{2}{3} \tilde{\rho} \mathcal{K} \nabla \cdot \overline{\mathbf{u}}+\mathrm{T}_{\mathrm{t}}: \nabla \overline{\mathbf{u}}-\frac{D_{\mathrm{t}} \tilde{\rho} \mathcal{K}^{3 / 2}}{L} \\
& -C_{\mathrm{B}} \frac{\mu_{\mathrm{t}}}{\tilde{\rho}^{2}}\left[\nabla \tilde{\rho}-(\nabla \tilde{\rho})_{\mathrm{ad}}\right] \cdot \nabla \tilde{P},
\end{aligned}
$$

where $(\nabla \tilde{\rho})_{\text {ad }}$ is the adiabatic density gradient (normally zero for non-stratified flows), and

$$
\mu_{\mathrm{t}}=A_{t} \tilde{\rho} L \mathcal{K}^{1 / 2}
$$

This transport equation is similar to one used by Deardorff [8] and in the familiar $k-\epsilon$ model $[9,10]$. All of these models have the shear creation term $T_{t}: \nabla \bar{u}$ in common.

We note in passing that we can derive a compressible generalization of the Smagorinsky eddy viscosity by assuming that viscous dissipation balances shear and buoyancy production in equation 14 . Then we algebraically eliminate $\mathcal{K}$ using equation 15 and $T_{t}$ by using equation 11 . The final result is

$$
\mu_{\mathrm{t}}=\left(\frac{A_{\mathrm{t}}^{3}}{D_{\mathrm{t}}}\right)^{1 / 2} \tilde{\rho} L^{2}\left\{\nabla \overline{\mathbf{u}}:\left[\nabla \overline{\mathbf{u}}+(\nabla \overline{\mathbf{u}})^{\mathrm{T}}-\frac{2}{3} \mathrm{U} \nabla \cdot \overline{\mathbf{u}}\right]-\frac{C_{\mathrm{B}}}{\tilde{\rho}^{2}}\left[\nabla \tilde{\rho}-(\nabla \tilde{\rho})_{\mathrm{ad}}\right] \cdot \nabla \tilde{P}\right\}^{1 / 2} .
$$

Comparing equations 12 and 16 , we see that

$$
C_{\mathrm{s}}^{2}=\left(\frac{A_{\mathrm{t}}^{3}}{D_{\mathrm{t}}}\right)^{1 / 2}
$$

In the remainder of this report, we shall drop the tildes and bars since the same numerical considerations will apply to both laminar and turbulent cases. 


\section{Expansion Into Components}

For numerical work, it is necessary to expand vector and tensor quantities into their components. We shall consider two coordinate systems: Cartesian coordinates and cylindrical coordinates. For the sake of easy reference, we shall summarize a number of differential operators in these two coordinate systems.

The gradient of a scalar is

$$
\begin{aligned}
& \nabla P=\frac{\partial P}{\partial x} \hat{x}+\frac{\partial P}{\partial y} \hat{y}+\frac{\partial P}{\partial z} \hat{z} \quad \text { Cartesian } \\
& \nabla P=\frac{\partial P}{\partial r} \hat{r}+\frac{1}{r} \frac{\partial P}{\partial \theta} \hat{\theta}+\frac{\partial P}{\partial z} \hat{z} \quad \text { Cylindrical }
\end{aligned}
$$

The divergence of the velocity is

$$
\begin{gathered}
\nabla \cdot \mathbf{u}=\frac{\partial u_{x}}{\partial x}+\frac{\partial u_{y}}{\partial y}+\frac{\partial u_{z}}{\partial z} \quad \text { Cartesian } \\
\nabla \cdot \mathbf{u}=\frac{1}{r} \frac{\partial\left(r u_{r}\right)}{\partial r}+\frac{1}{r} \frac{\partial u_{\theta}}{\partial \theta}+\frac{\partial u_{z}}{\partial z} \quad \text { Cylindrical }
\end{gathered}
$$

The advection of velocity may be expanded

$$
\begin{gathered}
\mathbf{u} \cdot \nabla \mathbf{u}=\left(u_{x} \frac{\partial u_{x}}{\partial x}+u_{y} \frac{\partial u_{x}}{\partial y}+u_{z} \frac{\partial u_{x}}{\partial z}\right) \hat{x}+\left(u_{x} \frac{\partial u_{y}}{\partial x}+u_{y} \frac{\partial u_{y}}{\partial y}+u_{z} \frac{\partial u_{y}}{\partial z}\right) \hat{y} \\
+\left(u_{x} \frac{\partial u_{z}}{\partial x}+u_{y} \frac{\partial u_{z}}{\partial y}+u_{z} \frac{\partial u_{z}}{\partial z}\right) \hat{z} \quad \text { Cartesian } \\
\mathbf{u} \cdot \nabla \mathbf{u}=\left(u_{r} \frac{\partial u_{r}}{\partial r}+\right. \\
\left.+\frac{u_{\theta}}{r} \frac{\partial u_{r}}{\partial \theta}-\frac{u_{\theta}^{2}}{r}+u_{z} \frac{\partial u_{r}}{\partial z}\right) \hat{r}+\left(u_{r} \frac{\partial u_{\theta}}{\partial r}+\frac{u_{\theta}}{r} \frac{\partial u_{\theta}}{\partial \theta}+\frac{u_{r} u_{\theta}}{r}+u_{z} \frac{\partial u_{\theta}}{\partial z}\right) \hat{\theta} \\
+\left(u_{r} \frac{\partial u_{z}}{\partial r}+\frac{u_{\theta}}{r} \frac{\partial u_{z}}{\partial \theta}+u_{z} \frac{\partial u_{z}}{\partial z}\right) \hat{z} \quad \text { Cylindrical }
\end{gathered}
$$

The present discussion makes extensive use of the velocity gradient tensor, which may be written in components as

$$
\begin{gathered}
\nabla \mathbf{u}=\left[\begin{array}{ccc}
\partial u_{x} / \partial x & \partial u_{y} / \partial x & \partial u_{z} / \partial x \\
\partial u_{x} / \partial y & \partial u_{y} / \partial y & \partial u_{z} \partial y \\
\partial u_{x} / \partial z & \partial u_{y} / \partial z & \partial u_{z} / \partial z
\end{array}\right] \quad \text { Cartesian } \\
\nabla \mathbf{u}=\left[\begin{array}{ccc}
\partial u_{r} / \partial r & \partial u_{\theta} / \partial r & \partial u_{z} / \partial r \\
r^{-1}\left(\partial u_{r} / \partial \theta-u_{\theta}\right) & r^{-1}\left(\partial u_{\theta} / \partial \theta+u_{r}\right) & r^{-1}\left(\partial u_{z} / \partial \theta\right) \\
\partial u_{r} / \partial z & \partial u_{\theta} / \partial z & \partial u_{z} / \partial z
\end{array}\right] \quad \text { Cylindrical }
\end{gathered}
$$

The components of the stress tensor $T$ in Cartesian coordinates are

$$
\tau_{x x}=\mu\left[2 \frac{\partial u_{x}}{\partial x}-\frac{2}{3} \nabla \cdot \mathbf{u}\right]+\mu_{\mathrm{b}} \nabla \cdot \mathbf{u}
$$




$$
\begin{gathered}
\tau_{y y}=\mu\left[2 \frac{\partial u_{y}}{\partial y}-\frac{2}{3} \nabla \cdot \mathbf{u}\right]+\mu_{\mathrm{b}} \nabla \cdot \mathbf{u} \\
\tau_{z z}=\mu\left[2 \frac{\partial u_{z}}{\partial z}-\frac{2}{3} \nabla \cdot \mathbf{u}\right]+\mu_{\mathrm{b}} \nabla \cdot \mathbf{u} \\
\tau_{x y}=\tau_{y x}=\mu\left[\frac{\partial u_{x}}{\partial y}+\frac{\partial u_{y}}{\partial x}\right] \\
\tau_{y z}=\tau_{z y}=\mu\left[\frac{\partial u_{y}}{\partial z}+\frac{\partial u_{z}}{\partial y}\right] \\
\tau_{z x}=\tau_{x z}=\mu\left[\frac{\partial u_{z}}{\partial x}+\frac{\partial u_{x}}{\partial z}\right]
\end{gathered}
$$

The components of the stress tensor $T$ in cylindrical coordinates are

$$
\begin{gathered}
\tau_{r r}=\mu\left[2 \frac{\partial u_{r}}{\partial r}-\frac{2}{3} \nabla \cdot \mathbf{u}\right]+\mu_{\mathrm{b}} \nabla \cdot \mathbf{u} \\
\tau_{\theta \theta}=\mu\left[2\left(\frac{1}{r} \frac{\partial u_{\theta}}{\partial \theta}+\frac{u_{r}}{r}\right)-\frac{2}{3} \nabla \cdot \mathbf{u}\right]+\mu_{\mathrm{b}} \nabla \cdot \mathbf{u} \\
\tau_{z z}=\mu\left[2 \frac{\partial u_{z}}{\partial z}-\frac{2}{3} \nabla \cdot \mathbf{u}\right]+\mu_{\mathrm{b}} \nabla \cdot \mathbf{u} \\
\tau_{r \theta}=\tau_{\theta r}=\mu\left[r \frac{\partial}{\partial r}\left(\frac{u_{\theta}}{r}\right)+\frac{1}{r} \frac{\partial u_{r}}{\partial \theta}\right] \\
\tau_{\theta z}=\tau_{z \theta}=\mu\left[\frac{\partial u_{\theta}}{\partial z}+\frac{1}{r} \frac{\partial u_{z}}{\partial \theta}\right] \\
\tau_{z r}=\tau_{r z}=\mu\left[\frac{\partial u_{z}}{\partial r}+\frac{\partial u_{r}}{\partial z}\right]
\end{gathered}
$$

The momentum equation contains the divergence of the stress tensor. Expanding in components and taking advantage of the symmetry of the stress tensor,

$$
\begin{gathered}
\nabla \cdot \mathrm{T}=\left(\frac{\partial \tau_{x x}}{\partial x}+\frac{\partial \tau_{x y}}{\partial y}+\frac{\partial \tau_{x z}}{\partial z}\right) \hat{x}+\left(\frac{\partial \tau_{x y}}{\partial x}+\frac{\partial \tau_{y y}}{\partial y}+\frac{\partial \tau_{y z}}{\partial z}\right) \hat{y} \\
+\left(\frac{\partial \tau_{x z}}{\partial x}+\frac{\partial \tau_{y z}}{\partial y}+\frac{\partial \tau_{z z}}{\partial z}\right) \hat{z} \quad \text { Cartesian } \\
\nabla \cdot \mathrm{T}=\left[\frac{1}{r} \frac{\partial\left(r \tau_{r r}\right)}{\partial r}+\frac{1}{r} \frac{\partial \tau_{r \theta}}{\partial \theta}-\frac{\tau_{\theta \theta}}{r}+\frac{\partial \tau_{r z}}{\partial z}\right] \hat{r}+\left[\frac{1}{r} \frac{\partial \tau_{\theta \theta}}{\partial \theta}+\frac{\partial \tau_{r \theta}}{\partial r}+\frac{2 \tau_{r \theta}}{r}+\frac{\partial \tau_{\theta z}}{\partial z}\right] \hat{\theta} \\
+\left[\frac{1}{r} \frac{\partial\left(r \tau_{r z}\right)}{\partial r}+\frac{1}{r} \frac{\partial \tau_{\theta z}}{\partial \theta}+\frac{\partial \tau_{z z}}{\partial z}\right] \hat{z} \quad \text { Cylindrical }
\end{gathered}
$$


The double dot product of any symmetric second-rank tensor represented by $T$ is given by the following relations:

$$
\begin{gathered}
\mathrm{T}: \nabla \mathbf{u}=\mathrm{T}: \mathrm{E}=\tau_{x x}\left(\frac{\partial u_{x}}{\partial x}\right)+\tau_{y y}\left(\frac{\partial u_{y}}{\partial y}\right)+\tau_{z z}\left(\frac{\partial u_{z}}{\partial z}\right) \\
+\tau_{x y}\left(\frac{\partial u_{x}}{\partial y}+\frac{\partial u_{y}}{\partial x}\right)+\tau_{y z}\left(\frac{\partial u_{y}}{\partial z}+\frac{\partial u_{z}}{\partial y}\right)+\tau_{z x}\left(\frac{\partial u_{z}}{\partial x}+\frac{\partial u_{x}}{\partial z}\right) \quad \text { Cartesian } \\
\mathrm{T}: \nabla \mathbf{u}=\tau_{r r}\left(\frac{\partial u_{r}}{\partial r}\right)+\tau_{\theta \theta}\left(\frac{1}{r} \frac{\partial u_{\theta}}{\partial \theta}+\frac{u_{r}}{r}\right)+\tau_{z z}\left(\frac{\partial u_{z}}{\partial z}\right) \\
+\tau_{r \theta}\left[r \frac{\partial}{\partial r}\left(\frac{u_{\theta}}{r}\right)+\frac{1}{r} \frac{\partial u_{r}}{\partial \theta}\right]+\tau_{\theta z}\left(\frac{1}{r} \frac{\partial u_{z}}{\partial \theta}+\frac{\partial u_{\theta}}{\partial z}\right)+\tau_{r z}\left(\frac{\partial u_{z}}{\partial r}+\frac{\partial u_{r}}{\partial z}\right) \quad \text { Cylindrical }
\end{gathered}
$$

If $T$ is the Newtonian stress tensor, these relations may be specialized to

$$
\begin{gathered}
\Phi \equiv \frac{1}{\mu} \mathrm{T}: \nabla \mathbf{u}=2\left[\left(\frac{\partial u_{x}}{\partial x}\right)^{2}+\left(\frac{\partial u_{y}}{\partial y}\right)^{2}+\left(\frac{\partial u_{z}}{\partial z}\right)^{2}\right] \\
+\left(\frac{\partial u_{x}}{\partial y}+\frac{\partial u_{y}}{\partial x}\right)^{2}+\left(\frac{\partial u_{y}}{\partial z}+\frac{\partial u_{z}}{\partial y}\right)^{2}+\left(\frac{\partial u_{z}}{\partial x}+\frac{\partial u_{x}}{\partial z}\right)^{2} \\
+\left(\frac{\mu_{\mathrm{b}}}{\mu}-\frac{2}{3}\right)\left[\frac{\partial u_{x}}{\partial x}+\frac{\partial u_{y}}{\partial y}+\frac{\partial u_{z}}{\partial z}\right]^{2} \text { Cartesian } \\
\Phi \equiv \frac{1}{\mu} \mathrm{T}: \nabla \mathbf{u}=2\left[\left(\frac{\partial u_{r}}{\partial r}\right)^{2}+\left(\frac{1}{r} \frac{\partial u_{\theta}}{\partial \theta}+\frac{u_{r}}{r}\right)^{2}+\left(\frac{\partial u_{z}}{\partial z}\right)^{2}\right] \\
+\left[r \frac{\partial}{\partial r}\left(\frac{u_{\theta}}{r}\right)+\frac{1}{r} \frac{\partial u_{r}}{\partial \theta}\right]^{2}+\left(\frac{1}{r} \frac{\partial u_{z}}{\partial \theta}+\frac{\partial u_{\theta}}{\partial z}\right)^{2}+\left(\frac{\partial u_{z}}{\partial r}+\frac{\partial u_{r}}{\partial z}\right)^{2} \\
+\left(\frac{\mu_{\mathrm{b}}}{\mu}-\frac{2}{3}\right)\left[\frac{1}{r} \frac{\partial\left(r u_{r}\right)}{\partial r}+\frac{1}{r} \frac{\partial u_{\theta}}{\partial \theta}+\frac{\partial u_{z}}{\partial z}\right]^{2} \text { Cylindrical }
\end{gathered}
$$

The COYOTE program solves the two dimensional Eulerian mean flow equations in both Cartesian and cylindrical coordinates. The differential equations to be solved are written in terms of the Cartesian coordinates $(x, z)$. For cylindrical coordinates, $x$ is the radial coordinate, and $z$ is the axial coordinate. Terms that appear only in cylindrical coordinates are multiplied by a parameter $\xi$, which is zero for Cartesian coordinates and unity for cylindrical coordinates. Similarly, it is convenient to introduce the variable $r$ which is unity in Cartesian coordinates, and $r=x$ in cylindrical coordinates.

The species continuity equations become

$$
\frac{\partial \rho_{\alpha}}{\partial t}+\frac{1}{r} \frac{\partial\left(r \rho_{\alpha} u\right)}{\partial x}+\frac{\partial\left(\rho_{\alpha} v\right)}{\partial z}=R_{\alpha}+\frac{1}{r} \frac{\partial}{\partial x}\left[r \rho D \frac{\partial}{\partial x}\left(\frac{\rho_{\alpha}}{\rho}\right)\right]+\frac{\partial}{\partial z}\left[\rho D \frac{\partial}{\partial z}\left(\frac{\rho_{\alpha}}{\rho}\right)\right],
$$


where $u$ and $v$ are the radial and axial components of the velocity, respectively. The total density is computed by summing the species densities.

The radial and axial momentum equations are

$$
\begin{gathered}
\rho\left(\frac{\partial u}{\partial t}+u \frac{\partial u}{\partial x}+v \frac{\partial u}{\partial z}\right)-\xi \frac{\rho w^{2}}{r}=\rho g_{x}-\frac{\partial P}{\partial x} \\
+\frac{1}{r} \frac{\partial}{\partial x}\left(2 r \mu \frac{\partial u}{\partial x}\right)+\frac{\partial}{\partial x}\left[\left(\mu_{\mathrm{b}}-\frac{2 \mu}{3}\right)\left(\frac{1}{r} \frac{\partial r u}{\partial x}+\frac{\partial v}{\partial z}\right)\right]+\frac{\partial}{\partial z}\left[\mu\left(\frac{\partial u}{\partial z}+\frac{\partial v}{\partial x}\right)\right]-\frac{2 \xi \mu u}{r^{2}}
\end{gathered}
$$

and

$$
\begin{gathered}
\rho\left(\frac{\partial v}{\partial t}+u \frac{\partial v}{\partial x}+v \frac{\partial v}{\partial z}\right)=\rho g_{z}-\frac{\partial P}{\partial z} \\
+\frac{1}{r} \frac{\partial}{\partial x}\left[r \mu\left(\frac{\partial u}{\partial z}+\frac{\partial v}{\partial x}\right)\right]+\frac{\partial}{\partial z}\left[2 \mu \frac{\partial v}{\partial z}+\left(\mu_{\mathrm{b}}-\frac{2 \mu}{3}\right)\left(\frac{1}{r} \frac{\partial r u}{\partial x}+\frac{\partial v}{\partial z}\right)\right]
\end{gathered}
$$

In cylindrical coordinates, we have the option of keeping all three velocity components, in which case the swirl (azimuthal) velocity $u_{\theta}=w$ is calculated from

$$
\frac{\partial \rho r w}{\partial t}+\frac{1}{r} \frac{\partial \rho r^{2} w u}{\partial x}+\frac{\partial \rho r w v}{\partial z}=\frac{1}{r} \frac{\partial}{\partial x}\left[r^{3} \mu \frac{\partial}{\partial x}\left(\frac{w}{r}\right)\right]+\frac{\partial}{\partial z}\left[\mu r^{2} \frac{\partial}{\partial z}\left(\frac{w}{r}\right)\right]
$$

This strong conservation form allows the finite difference equation to exactly conserve the axial component of angular momentum, $r w$.

The internal energy equation is

$$
\begin{aligned}
& \frac{\partial \rho I}{\partial t}+\frac{1}{r} \frac{\partial r \rho I u}{\partial x}+\frac{\partial \rho I v}{\partial z}=-P\left(\frac{1}{r} \frac{\partial r u}{\partial x}+\frac{\partial v}{\partial z}\right)+\left[\frac{1}{r} \frac{\partial}{\partial x}\left(r K \frac{\partial T}{\partial x}\right)+\frac{\partial}{\partial z}\left(K \frac{\partial T}{\partial z}\right)\right] \\
&+\mu\left\{2\left(\frac{\partial u}{\partial x}\right)^{2}+2\left(\frac{\partial v}{\partial z}\right)^{2}+\left(\frac{\partial u}{\partial z}+\frac{\partial v}{\partial x}\right)^{2}+\frac{2 \xi u^{2}}{x^{2}}+\xi\left[r \frac{\partial}{\partial x}\left(\frac{w}{r}\right)\right]^{2}+\xi\left(\frac{\partial w}{\partial z}\right)^{2}\right\} \\
&+\left(\mu_{\mathrm{b}}-\frac{2 \mu}{3}\right)\left(\frac{1}{r} \frac{\partial(r u)}{\partial x}+\frac{\partial v}{\partial z}\right)^{2} \\
&+\sum_{\alpha}\left\{H_{\alpha} R_{\alpha}+\frac{1}{r} \frac{\partial}{\partial x}\left[r h_{\alpha} \rho D \frac{\partial}{\partial x}\left(\frac{\rho_{\alpha}}{\rho}\right)\right]+\frac{\partial}{\partial z}\left[h_{\alpha} \rho D \frac{\partial}{\partial z}\left(\frac{\rho_{\alpha}}{\rho}\right)\right]\right\}
\end{aligned}
$$

where the conductivity $K$ is given by

$$
K=\mu C_{P} / \operatorname{Pr}
$$

where $\operatorname{Pr}$ is the Prandtl number and $C_{P}$ is the specific heat at constant pressure. 


\section{Numerical Considerations}

The viscous dissipation term $T: \nabla \mathbf{u}$ in the $I$ equation and the terms of the same form in the turbulence models can be differenced in a variety of ways. In this section, we shall use the COYOTE combustion code [1] to provide examples of how the choice of differencing methods can affect the accuracy of the solutions even on fine grids. Crudely speaking, this behavior is due to the possibility of a formally second-order truncation error behaving essentially as a zeroth-order error in cases where grid-scale flow features remain as the grid is refined (which is the situation in LES). This effect will be discussed in a later subsection on idealized flows

where we shall find an inconsistency between the momentum and energy equations for one of the difference methods.

COYOTE is an Eulerian code based on a rectangular staggered grid. Velocity components are defined on cell faces, and all other quantities are defined at cell centers, the same as in the original ICE method [11] and in SOLA-ICE [12]. COYOTE has the options for twoand three-dimensional Cartesian coordinates and two-dimensional cylindrical coordinates. Although the last option assumes axial symmetry, it retains all three velocity components so we can simulate swirling flows.

This section provides a detailed description of the grid and selected aspects of the finite difference methods used in the COYOTE program to approximate the viscous terms. A fragment of a typical computational grid is illustrated in figure 1. The Eulerian mesh comprises rectangular cells having variable sizes, $\delta x_{i}$ for the $i^{\text {th }}$ column and $\delta z_{j}$ for the $j^{\text {th }}$ row. The dependent variables are located within a cell as follows: the horizontal (radial) velocity component $u$ is defined at the middle of the vertical sides, the vertical (axial) component $v$ at the middle of the horizontal sides, and all other variables at the cell centers. Subscripts $i$ and $j$ denote cell centers, $i+1 / 2$ denotes the right cell edge, and $j+1 / 2$ denotes the upper cell edge.

The mesh is defined in the $x$-direction by specifying the locations of cell edges $x_{i-1 / 2}$. Cell centers are then computed as

$$
x_{i}=0.5\left(x_{i-1 / 2}+x_{i+1 / 2}\right)
$$

To maintain at least first order spatial accuracy on a nonuniform mesh, some attention must be paid to the centering of the finite difference terms. In some instances, the average position of two cell centers,

$$
\bar{x}_{i-1 / 2}=0.5\left(x_{i-1}+x_{i}\right)
$$

will be required instead of the cell edge position. The mesh in the $z$-direction is defined in an analogous fashion. 
The reason for this particular form of staggered grid is to minimize a particular parasitic mode often called "hourglassing." Hourglassing is characterized by a strong everyother-zone oscillatory component of the velocity field, e.g. $u\left(x_{i+1 / 2}, z_{j}, t\right)=(-1)^{i+j}$. This mode will occur naturally in large eddy simulations or direct numerical simulations where the numerical resolution is barely adequate to capture the smallest flow features. It can also be a highly undesirable numerical artifact. Hourglassing problems frequently are due to difference methods that are "blind" to this mode, failing to produce physically correct damping or restoring forces. In methods which locate all flow variables at the same points, this mode can arise from numerical differentiation across more than one zone at a time. A good example is the FLIC method [13], in which the pressure gradient at a cell center is independent of the pressure there, producing a tendency for this mode to occur (plus causing unconditional instability for flows below Mach 0.1 or so, just for good measure).

In methods which locate all velocity components at cell corners and everything else at cell centers (commonly used in Lagrangian codes), this mode can be especially difficult to control. If the pressure field has an every-other-zone perturbation, which tends to accompany the velocity oscillations, pressure gradients produce forces with the same magnitude but alternating signs on alternate cell faces, which are then averaged to produce a zero correcting force at the cell corners. The cure is to change to a differencing method that does produce the appropriate restoring or damping forces. This is preferable to the ad hoc procedures such as the node coupler $[14,15,16]$ sometimes employed. The CONCHAS-SPRAY 2-D arbitrary Lagrangian-Eulerian (ALE) program [15] attempted to fix this problem by introducing an eight-sided momentum control volume, but this approach was only partially successful. A more successful approach was used in an early version of the KIVA 3D ALE code in which the corner velocities were interpolated to produce cell-face-centered velocities at selected points in the calculations [17].

What we discovered in COYOTE is that the orginal method [1] uses a difference method for the viscous dissipation terms that is blind to the hourglassing mode, producing zero dissipation, even though this mode has a large rate of strain that produces corrective forces in the momentum equation. In the remainder of this section, we shall examine the differencing of all the viscous terms and recommend a new scheme for the dissipation term in the internal energy equation.

Let us begin by considering the differencing of the viscous terms in the axial momentum equation. Similar considerations and conclusions apply to the radial momentum equation as well.

First, consider the differencing of the velocity divergence.

$$
(\nabla \cdot \mathbf{u})_{i, j}=\frac{r_{i+1 / 2} u_{i+1 / 2, j}-r_{i-1 / 2} u_{i-1 / 2, j}}{r_{i}\left(x_{i+1 / 2}-x_{i-1 / 2}\right)}+\frac{v_{i, j+1 / 2}-v_{i, j-1 / 2}}{z_{j+1 / 2}-z_{j-1 / 2}} .
$$


As we can see from figure 1 , the velocity divergence is naturally centered on cell centers $(i, j)$. This approximation is second-order and has no known pathological numerical behavior (note that $r_{i}$ is always off the axis in cylindrical coordinates, so there is never an issue with division by zero).

Now consider the differencing of the viscous terms by referring to figure 2, which shows a fragment of the grid with the axial momentum control volume for $v_{i, j-1 / 2}$ shown by dashed lines. The derivatives in the vertical direction are, neglecting the bulk viscosity,

$$
\begin{gathered}
\left\{\frac{\partial}{\partial z}\left[2 \mu \frac{\partial v}{\partial z}-\frac{2 \mu}{3}\left(\frac{1}{r} \frac{\partial r u}{\partial x}+\frac{\partial v}{\partial z}\right)\right]\right\}_{i, j-1 / 2}= \\
\frac{2}{z_{j}-z_{j-1}}\left[\mu_{i, j}\left(\frac{v_{i, j+1 / 2}-v_{i, j-1 / 2}}{z_{j+1 / 2}-z_{j-1 / 2}}-\frac{1}{3}(\nabla \cdot \mathbf{u})_{i, j}\right)-\mu_{i, j-1}\left(\frac{v_{i, j-1 / 2}-v_{i, j-3 / 2}}{z_{j-1 / 2}-z_{j-3 / 2}}-\frac{1}{3}(\nabla \cdot \mathbf{u})_{i, j-1}\right)\right] .
\end{gathered}
$$

Notice that the viscosities and velocity divergences are needed at cell centers, where they are naturally defined. The terms containing $v$ are a standard three-point centered scheme in each direction, which correctly produces damping forces for the hourglassing mode.

On the other hand, the radial derivative term requires evaluation of $\tau_{x z}$ on the cell corners denoted by open circles in figure 2 .

$$
\left\{\frac{1}{r} \frac{\partial}{\partial x}\left[r \mu\left(\frac{\partial u}{\partial z}+\frac{\partial v}{\partial x}\right)\right]\right\}_{i, j-1 / 2}=\frac{\bar{r}_{i+1 / 2}\left(\tau_{x z}\right)_{i+1 / 2, j-1 / 2}-\bar{r}_{i-1 / 2}\left(\tau_{x z}\right)_{i-1 / 2, j-1 / 2}}{r_{i}\left(\bar{x}_{i+1 / 2}-\bar{x}_{i-1 / 2}\right)} .
$$

We next calculate the $x z$ component of the stress tensor at cell corners,

$$
\left(\tau_{x z}\right)_{i-1 / 2, j-1 / 2}=\mu_{i-1 / 2, j-1 / 2}\left(\frac{u_{i-1 / 2, j}-u_{i-1 / 2, j-1}}{z_{j}-z_{j-1}}+\frac{v_{i, j-1 / 2}-v_{i-1, j-1 / 2}}{x_{i}-x_{i-1}}\right) .
$$

An average of four cell-centered viscosities is taken for the cell-corner viscosity. ${ }^{1}$ The required velocity components are defined just where they are needed, so no averaging of them is required. Again, the $v$ part of this scheme will smooth the hourglass mode. Similar good results are obtained for the $u$ velocity component and the swirl velocity. Averaging or

\footnotetext{
${ }^{1}$ One could improve upon this approximation by interpolating the cell-centered viscosities onto the cell corners, but this extra computational effort is unlikely to produce a significant improvement in accuracy since we always use a grid that is nearly uniform with adjacent cell sizes varying by no more than a few percent. In the same vein, we should note that the two velocity derivatives in equation 45 are defined at $\left(x_{i-1 / 2}, \bar{z}_{j-1 / 2}\right)$ and $\left(\bar{x}_{i-1 / 2}, z_{j-1 / 2}\right)$ rather than at the true cell corner $\left(x_{i-1 / 2}, z_{j-1 / 2}\right)$. To insure formal first order accuracy, we should treat the two derivatives separately to take this fact into account. That is, we should interpolate the viscosities to both of these points, apply the different values to their corresponding velocity derivatives, and use $\left(x_{i+1 / 2}-x_{i-1 / 2}\right)$ instead of $\left(\bar{x}_{i+1 / 2}-\bar{x}_{i-1 / 2}\right)$ for the $u$ term in equation 44 . Since these changes are not expected to make much difference in the final analysis, we have chosen the computationally simpler scheme shown here. The use of $\bar{x}$ in equation 44 makes the $v$-diffusion terms first order (except for the averaging of the viscosity) in the $v$ momentum equation. A future version of the code will contain these modifications in the interest of insuring a consistent difference method on a nonuniform grid, even though in practice the changes are not expected to produce much difference.
} 
interpolation is required only for the viscosity itself, which has never been known to introduce a difficulty.

The situation for the viscous dissipation in the energy equation is less felicitous. The problem is that we need things that are defined at cell centers, which some of them are. But there are others that are naturally defined at cell corners. The original differencing scheme is

$$
\begin{gathered}
\Phi_{i, j} \equiv \frac{1}{\mu_{i, j}}(\mathbf{T}: \nabla \mathbf{u})_{i, j}=\left[2\left(\frac{\partial u}{\partial x}\right)^{2}+2\left(\frac{\partial v}{\partial z}\right)^{2}+\left(\frac{\mu_{\mathrm{b}}}{\mu}-\frac{2}{3}\right)(\nabla \cdot \mathbf{u})^{2}+\left(\frac{\partial u}{\partial z}+\frac{\partial v}{\partial x}\right)^{2}\right. \\
\left.+2 \xi\left(\frac{u}{r}\right)^{2}+\xi\left(\frac{\partial w}{\partial x}-\frac{w}{r}\right)^{2}+\xi\left(\frac{\partial w}{\partial z}\right)^{2}\right]_{i, j} \\
=2\left(\frac{u_{i+1 / 2, j}-u_{i-1 / 2, j}}{x_{i+1 / 2}-x_{i-1 / 2}}\right)^{2}+2\left(\frac{v_{i, j+1 / 2}-v_{i, j-1 / 2}}{z_{j+1 / 2}-z_{j-1 / 2}}\right)^{2}+\left(\frac{\mu_{\mathrm{b}, i, j}}{\mu_{i, j}}-\frac{2}{3}\right)(\nabla \cdot \mathbf{u})_{i, j}^{2} \\
+\left[\frac{u_{i+1 / 2, j+1}+u_{i-1 / 2, j+1}-u_{i+1 / 2, j-1}-u_{i-1 / 2, j-1}}{2\left(z_{j+1}-z_{j-1}\right)}\right. \\
\left.+\frac{v_{i+1, j+1 / 2}+v_{i+1, j-1 / 2}-v_{i-1, j+1 / 2}-v_{i-1, j-1 / 2}}{2\left(x_{i+1}-x_{i-1}\right)}\right]^{2} \\
+\frac{\xi\left(u_{i+1 / 2, j}+u_{i-1 / 2, j}\right)^{2}}{2 x_{i}^{2}}+\xi\left(\frac{w_{i+1, j}-w_{i-1, j}}{x_{i+1}-x_{i-1}}-\frac{w_{i, j}}{x_{i}}\right)^{2}+\xi\left(\frac{w_{i, j+1}-w_{i, j-1}}{z_{j+1}-z_{j-1}}\right)^{2} .
\end{gathered}
$$

Note that the diagonal terms are evaluated with differences across a single zone, so the hourglassing mode produces dissipation. As can be seen from figure 3, the off-diagonal term, as well as most of the cylindrical and swirl terms, involve either averaging of two neighboring velocity values or differences over two zones. In both cases, the dissipation produced by the hourglass mode is zero, a nonphysical result.

To produce a more realistic approximation, let us first consider the off-diagonal term evaluated at corner 3 as illustrated in figure 4 .

$$
\left(\tau_{x z}\right)_{i+1 / 2, j+1 / 2}=\left(\frac{\partial u}{\partial z}+\frac{\partial v}{\partial x}\right)_{i+1 / 2, j+1 / 2}^{2}=\left(\frac{u_{i+1 / 2, j+1}-u_{i+1 / 2, j}}{z_{j+1}-z_{j}}+\frac{v_{i+1, j+1 / 2}-v_{i, j+1 / 2}}{x_{i+1}-x_{i}}\right)^{2}
$$

This will produce dissipation for the hourglass mode, but it is defined on corner 3 , not the cell center $(i, j)$. However, if we evaluate this term on all four corners and average the four positive numbers, we will get a positive dissipation rate at the cell center.

Similar considerations apply also to the swirl terms, which we shall define on two cell faces and average. The $u / r$ term cannot be evaluated this way since $r_{i-1 / 2}=0$ on the axis. So, we shall have to live with the present differencing of this term. Pulling it all together,

$$
\Phi_{i, j}=2\left(\frac{u_{i+1 / 2, j}-u_{i-1 / 2, j}}{x_{i+1 / 2}-x_{i-1 / 2}}\right)^{2}+2\left(\frac{v_{i, j+1 / 2}-v_{i, j-1 / 2}}{z_{j+1 / 2}-z_{j-1 / 2}}\right)^{2}+\left(\frac{\mu_{\mathrm{b}, i, j}}{\mu_{i, j}}-\frac{2}{3}\right)(\nabla \cdot \mathbf{u})_{i, j}^{2}
$$




$$
\begin{gathered}
+\frac{1}{4}\left[\left(\tau_{x z}\right)_{i-1 / 2, j-1 / 2}^{2}+\left(\tau_{x z}\right)_{i+1 / 2, j-1 / 2}^{2}+\left(\tau_{x z}\right)_{i+1 / 2, j+1 / 2}^{2}+\left(\tau_{x z}\right)_{i-1 / 2, j+1 / 2}^{2}\right] \\
+\frac{\xi\left(u_{i+1 / 2, j}+u_{i-1 / 2, j}\right)^{2}}{2 x_{i}^{2}}+\frac{\xi}{2}\left\{\left[\frac{\bar{r}_{i+1 / 2}}{x_{i+1}-x_{i}}\left(\frac{w_{i+1, j}}{r_{i+1}}-\frac{w_{i, j}}{r_{i}}\right)\right]^{2}+\left[\frac{r_{i-1 / 2}}{x_{i}-x_{i-1}}\left(\frac{w_{i, j}}{r_{i}}-\frac{w_{i-1, j}}{r_{i-1}}\right)\right]^{2}\right\} \\
+\frac{\xi}{2}\left[\left(\frac{w_{i, j+1}-w_{i, j}}{z_{j+1}-z_{j}}\right)^{2}+\left(\frac{w_{i, j}-w_{i, j-1}}{z_{j}-z_{j-1}}\right)^{2}\right]
\end{gathered}
$$

Now let us consider the truncation errors associated with these two difference methods applied to $\Phi$ in Cartesian coordinates. Using the usual Taylor series expansion technique (for example, $[18,19]$; see also [20]), we find that both are second order in space on a uniform grid. For the original method, the off-diagonal terms give

$$
\begin{gathered}
\left(\frac{\partial u}{\partial z}+\frac{\partial v}{\partial x}\right)_{i, j}^{2}=\left(\frac{\partial u}{\partial z}+\frac{\partial v}{\partial x}\right)^{2}+\frac{\delta x^{2}}{3}\left(\frac{\partial u}{\partial z}+\frac{\partial v}{\partial x}\right)\left(\frac{\partial^{3} u}{\partial z \partial x^{2}}+\frac{\partial^{3} v}{\partial x^{3}}\right) \\
+\frac{\delta z^{2}}{3}\left(\frac{\partial u}{\partial z}+\frac{\partial v}{\partial x}\right)\left(\frac{\partial^{3} u}{\partial z^{3}}+\frac{\partial^{3} v}{\partial x \partial z^{2}}\right)+\mathcal{O}(4) .
\end{gathered}
$$

For the new method, the expansion yields

$$
\begin{gathered}
\left(\frac{\partial u}{\partial z}+\frac{\partial v}{\partial x}\right)_{i, j}^{2}=\left(\frac{\partial u}{\partial z}+\frac{\partial v}{\partial x}\right)^{2}+\delta x^{2}\left[\left(\frac{\partial^{2} u}{\partial x \partial z}+\frac{\partial^{2} v}{\partial x^{2}}\right)^{2}+\left(\frac{\partial u}{\partial z}+\frac{\partial v}{\partial x}\right)\left(\frac{1}{4} \frac{\partial^{3} u}{\partial z \partial x^{2}}+\frac{1}{3} \frac{\partial^{3} v}{\partial x^{3}}\right)\right] \\
+\delta z^{2}\left[\left(\frac{\partial^{2} u}{\partial z^{2}}+\frac{\partial^{2} v}{\partial x \partial z}\right)^{2}+\left(\frac{\partial u}{\partial z}+\frac{\partial v}{\partial x}\right)\left(\frac{1}{3} \frac{\partial^{3} u}{\partial z^{3}}+\frac{1}{4} \frac{\partial^{3} v}{\partial x \partial z^{2}}\right)\right]+\mathcal{O}(4)
\end{gathered}
$$

Here the term $\mathcal{O}(4)$ denotes truncation error terms of order 4 and higher. ${ }^{2}$

\section{Numerical Examples}

It is difficult to assess the accuracy of a numerical method without running multiple test problems. This section presents two tests of our differencing of $\Phi$ : a pair of simple flow fields with known solutions and a turbulent Rayleigh-Taylor instability. The former illustrates how simple idealized situations may be used to check for correct behavior in certain limits. The latter shows one example of a typical CFD problem where the details of the differencing make a small but not necessarily negligible effect.

\footnotetext{
${ }^{2}$ As noted in another footnote, some of the averages must be replaced by interpolation to insure at least first order accuracy on a nonuniform grid. This is important since zeroth order errors mean the method is not consistent, and therefore cannot be convergent except on a uniform grid.
} 


\subsection{Idealized Flows}

First we look at the general case in two-dimensional Cartesian coordinates of rigid-body rotation about the point $\left(x_{0}, z_{0}\right):(u, v)=\omega\left(-z+z_{0}, x-x_{0}\right)$ where $\omega$ is the constant rotation rate. Analytically, the velocity gradient is antisymmetric, so the rate of strain tensor is zero, and therefore $\Phi$ is zero. It is easy to verify by direct substitution that both difference methods give a value of $\Phi=0$. This is to be expected since the truncation errors in both cases are proportional to second and higher derivatives, all of which are identically zero.

The second case is more relevant to LES and direct numerical simulations (DNS), where flow features occur at the limits of grid resolution. Consider the two every-other-cell modes that flip-flop in both directions. We consider the case of two-dimensional Cartesian coordinates on a uniform grid with zones of size $\delta x$ by $\delta z$. The velocity components are

$$
u_{i-1 / 2, j}= \pm(-1)^{i+j} \epsilon \delta x
$$

and

$$
v_{i, j-1 / 2}=(-1)^{i+j} \epsilon \delta z
$$

where $\epsilon$ is an arbitrary constant.

If we take the minus sign in equation 51, both $u$ components flow into (out of) a given zone, while both $v$ components flow out of (into) it. This flow pattern produces circulation around each corner, with opposite direction of flow around neighboring corners. This flow field approximates a rectangular array of counter-rotating vortices at the finest resolution that can be resolved on this grid regardless of which numerical method is being used. If we substitute this velocity field into the new difference method, we obtain

$$
\Phi_{i, j}=16 \epsilon^{2}+4 \epsilon^{2}\left(\frac{\delta x}{\delta z}-\frac{\delta z}{\delta x}\right)^{2} .
$$

The old difference method gives only $16 \epsilon^{2}$, which is the same as the new method only if $\delta x=\delta z$. So, which is more reasonable? We can calculate the finite difference dissipation rate due to the finite difference viscous forces very easily. The heat generation rate is just the negative of the viscous force dotted into the velocity vector, so for a given cell, we can calculate the viscous force on each cell face from the momentum difference equation and multiply by the negative of the velocity perpendicular to that face. If we apportion this heat production rate evenly to the two zones on either side of the face, then we find the dissipation rate expected from the momentum equations is just equation 53 if the viscosity is constant. This means our new difference equation for the dissipation rate is more internally consistent unless the zones are square. 
If we take the plus sign in equation 51 , all four velocity components on the faces of a given cell are directed either inward or outward. For this compressive mode, the new difference method predicts a dissipation rate of

$$
\Phi_{i, j}=16 \epsilon^{2}\left(\frac{1}{3}+\frac{\mu_{\mathrm{b}}}{\mu}\right)+4 \epsilon^{2}\left(\frac{\delta z}{\delta x}+\frac{\delta x}{\delta z}\right)^{2} .
$$

As in the previous case, the term involving the zone size is missing for the old method. Again doing the exercise of estimating the viscous forces from the momentum difference equations, we find the new method produces the expected answer. This time the old method is inconsistent even for square zones.

We note that this procedure of analyzing the behavior of numerical methods applied to the every-other-zone modes is a general and powerful tool for detecting certain types of pathological behavior.

\subsection{Rayleigh-Taylor Instabilities}

We ran a simulation of a generic Rayleigh-Taylor instability with the COYOTE computational fluid dynamics program [1]. The appendix contains the input file. The turbulence model is the Smagorinsky model as presented by Deardorff [5] with a Smagorinsky constant of 0.176 . The grid is $10 \mathrm{~cm}$ wide by $40 \mathrm{~cm}$ tall with square zones $0.5 \mathrm{~mm}$ on each side. Fluids with densities 0.0157 and $0.01 \mathrm{~g} / \mathrm{cm}^{3}$ (Atwood number 0.222 ) are subjected to a gravitational field of $6.86 \times 10^{4} \mathrm{~cm} / \mathrm{s}^{2}(70 \mathrm{G})$. The fluids are both at $T=287.7 \mathrm{~K}$, have molecular weights of 15.7 and 10.0, and both have $\gamma=5 / 3$. Molecular transport coefficients were computed from the Lennard-Jones parameters for helium and $\mathrm{N}_{2}$, which were arbitrarily chosen since molecular diffusion is small in this problem. Selected plots of mass fraction contours are shown in figures 5-10.

Figure 5 shows the mass fraction contours for the heavy species in the initial condition. The interface is in the middle of the grid and has a single-mode perturbation with a wavelength of $10 \mathrm{~cm}$ and peak-to-peak amplitude $0.8 \mathrm{~cm}$. The same initial conditions were used for both the old and new differencing.

Figures 6 and 7 show the solutions at $t=50 \mathrm{~ms}$. At this time, the instability has become nonlinear, although it is still basically laminar. All flow features are well resolved, and there is almost no difference between the results using the old and new differencing methods. At this early time, this is not the ideal test problem for testing difference methods for the viscous dissipation because at the low Mach number of this flow, the viscous dissipation term and the eddy viscosity are quite small until quite late in the problem.

However, we do see differences when the turbulence becomes well developed and the eddy viscosity becomes significant. Figures 8 and 9 show the solutions at $t=110 \mathrm{~ms}$. Now 
secondary instabilities are quite pronounced, and the heavy fluid has almost reached the bottom boundary. Although the overall growth rate of the thickness of the mixed layer is the same in both cases, there are many differences in the small details of the flow. This is to be expected since the flow is now quite turbulent and the exponential growth of small disturbances (the so-called "butterfly effect") should be seen. The differences between the two solutions show up in other quantities as well as in the density field. For example, the maximum fluid speed is $v_{\max }=119.5 \mathrm{~cm} / \mathrm{s}$ with old method, $98.5 \mathrm{~cm} / \mathrm{s}$ with the new. With the old method, kinematic viscosity ranges from 0.012 to $1.67 \mathrm{~cm}^{2} / \mathrm{s}$. With the new method, 0.012 to $1.34 \mathrm{~cm}^{2} / \mathrm{s}$. The lowest value is the molecular viscosity, and larger values reflect the addition of the Smagorinsky eddy viscosity.

Which solution is better? And in what sense? It is hard to be certain since there is no suitable experimental data for this problem. However, I would choose the new method because of its qualitatively better behavior at the limit of resolution as explained in the previous subsection. A more stringent test would require comparison of probability distribution functions, turbulent spectra, or other statistical quantities with a true DNS or relevant experimental data, none of which are available.

As a side note, Figure 10 shows the mass fraction contours for the new case at 110 ms without a turbulence model. Here we see a quite different solution. The Smagorinsky model makes a difference, even for this low-order code and good resolution (200 by 800 grid) of the intial stages of the instability. Even at $50 \mathrm{~ms}$, this pseudo-DNS is showing secondary vortices that are suppressed by the turbulence model. By $110 \mathrm{~ms}$, as shown in the figure, the flow is quite different from the LES cases. The lesson is quite clear: one cannot rely on the numerical dissipation, even with a second-order method, to supply sufficient dissipation to mimic the effects of a reasonable eddy viscosity model. To do so is poor CFD practice as it amounts to an uncontrolled approximation of important physical processes.

\section{Summary}

The hourglass, or every-other-cell, modes are problematic for many finite difference computational fluid dynamics methods and great care must be taken to avoid pathologies in the solutions. We show an example where these modes can cause an inconsistency between the momentum and energy equations on a rectangular staggered grid that was intended to minimize such difficulties. These modes are the shortest Fourier modes that can be resolved (admittedly poorly), and they will tend to be the worst-behaved with respect to stability and accuracy issues. This propensity can be turned to advantage by using analysis of the behavior of these modes as one test of the desirability of any particular difference scheme.

We compared two difference methods for the double dot product of the velocity gra- 
dient and rate of strain tensors, which occurs in the viscous dissipation term and in many turbulence models. Both methods are formally second order accurate, but one of them was shown to be inconsistent with the momentum equation in the limiting case of the everyother-cell modes. The recommended difference method produces the correct dissipation rate for these short modes. We note that Deardorff [5] uses the more consistent differencing method to evaluate the Smagorinsky eddy viscosity.

Simulations of a Rayleigh-Taylor instability showed that the turbulence model produced noticable effects even early in the problem where the flow is beginning its transition from laminar flow to turbulence. This result suggests that a model specifically designed to treat low-intensity turbulence must be included in studies of Rayleigh-Taylor instabilities. A little reflection suggests that such a model will also be required in the case of RichtmyerMeshkov instabilities. The effects of the turbulence model grow as the instability progresses and becomes more turbulent, and the differences between the two difference methods become noticable. Although we lack the relevant experimental data to determine which difference method is the actually the better, we recommend the newer, more consistent one.

\section{Acknowledgements}

This work was performed under the auspices of the U. S. Department of Energy by University of California Lawrence Livermore National Laboratory under contract No. W-7405-Eng-48.

\section{References}

[1] L. D. Cloutman, "COYOTE: A computer program for 2D reactive flow simulations," Lawrence Livermore National Laboratory Report UCRL-ID-103611, 1990.

[2] R. B. Bird, W. E. Stewart, and E. N. Lightfoot, Transport Phenomena (Wiley, New York, 1960).

[3] S. Chapman and T. G. Cowling, The Mathematical Theory of Non-Uniform Gases (Cambridge University Press, London, 1953).

[4] J. Smagorinsky, "General circulation experiments with the primitive equations I. The basic experiment," Mon. Weather Rev. 91, 99 (1963).

[5] J. W. Deardorff, "On the magnitude of the subgrid scale eddy coefficient," J. Comput. Phys. 7, 120 (1971). 
[6] L. D. Cloutman. 1991, "The LUVD11 large eddy simulation model," (Lawrence Livermore National Lab. Rept. UCRL-ID-107128).

[7] L. D. Cloutman, "Numerical simulations of the heat transfer and decay of turbulent swirling flows," Computers \& Fluids 17, 437 (1989).

[8] J. W. Deardorff, "The use of subgrid transport equations in a three-dimensional model of atmospheric turbulence," J. Fluids Eng., 95, 429 (1973).

[9] W. P. Jones and B. E. Launder, "The prediction of laminarization with a two-equation model of turbulence," Int. J. Heat Mass Transfer, 15, 301 (1972).

[10] W. P. Jones, in Prediction Methods for Turbulent Flows, ed. W. Kollmann (Hemisphere, Washington, 1980), p. 379.

[11] F. H. Harlow and A. A. Amsden, "A numerical fluid dynamics calculation method for all flow speeds," J. Comput. Phys. 8, 197 (1971).

[12] L. D. Cloutman, C. W. Hirt, and N. C. Romero, "SOLA-ICE: A numerical solution algorithm for transient compressible fluid flows," Los Alamos National Laboratory report LA-6236-MS (1976).

[13] R. A. Gentry, R. E. Martin, and B. J. Daly, "An Eulerian differencing method for unsteady compressible flow problems," J. Comput. Phys, 1, 87 (1966).

[14] C. W. Hirt, A. A. Amsden, and J. L. Cook, "An arbitrary Lagrangian-Eulerian computing method for all flow speeds," J. Comput. Phys. 14, 227 (1974).

[15] L. D. Cloutman, J. K. Dukowicz, J. D. Ramshaw, and A. A. Amsden, "CONCHASSPRAY: A computer code for reactive flows with fuel sprays," Los Alamos National Laboratory report LA-9294-MS (1982).

[16] A. A. Amsden, J. D. Ramshaw, P. J. O'Rourke, and J. K. Dukowicz, "KIVA: A computer program for two- and three-dimensional fluid flows with chemical reactions and fuel sprays," Los Alamos National Laboratory report LA-10245-MS (1985).

[17] A. A. Amsden, J. D. Ramshaw, L. D. Cloutman, and P. J. O'Rourke, "Improvements and extensions to the KIVA computer program," Los Alamos National Laboratory report LA-10534-MS (1985).

[18] C. W. Hirt, "Heuristic stability theory for finite-difference equations," J. Comput. Phys. 2, 339 (1968). 
[19] R. F. Warming and B. J. Hyett, "The modified equation approach to the stability and accuracy analysis of finite-difference methods," J. Comput. Phys. 14, 159 (1974).

[20] S.-C. Chang, "A critical analysis of the modified equation technique of Warming and Hyett," J. Comput. Phys. 86, 107 (1990). 


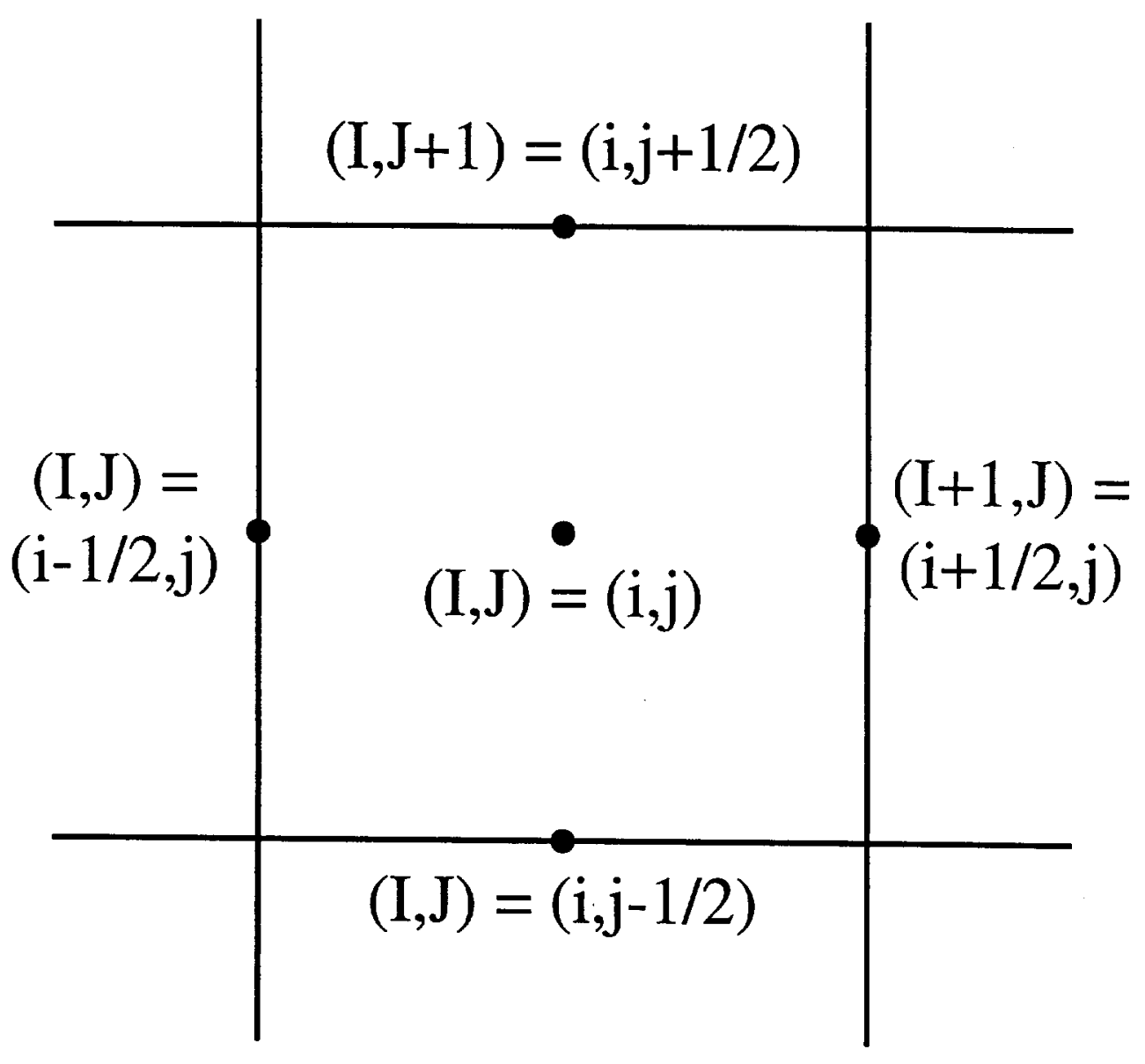

Figure 1: A fragment of the staggered COYOTE grid. Horizontal velocity $u_{x}=u$ is defined at the centers of the vertical cell faces $(i \pm 1 / 2, j)$, and vertical velocity $u_{z}=v$ is defined at the centers of the horizontal cell faces $(i, j \pm 1 / 2)$. All other quantities are defined at cell centers $(i, j)$. Capitalized letters denote the Fortran indices as used in the program. 


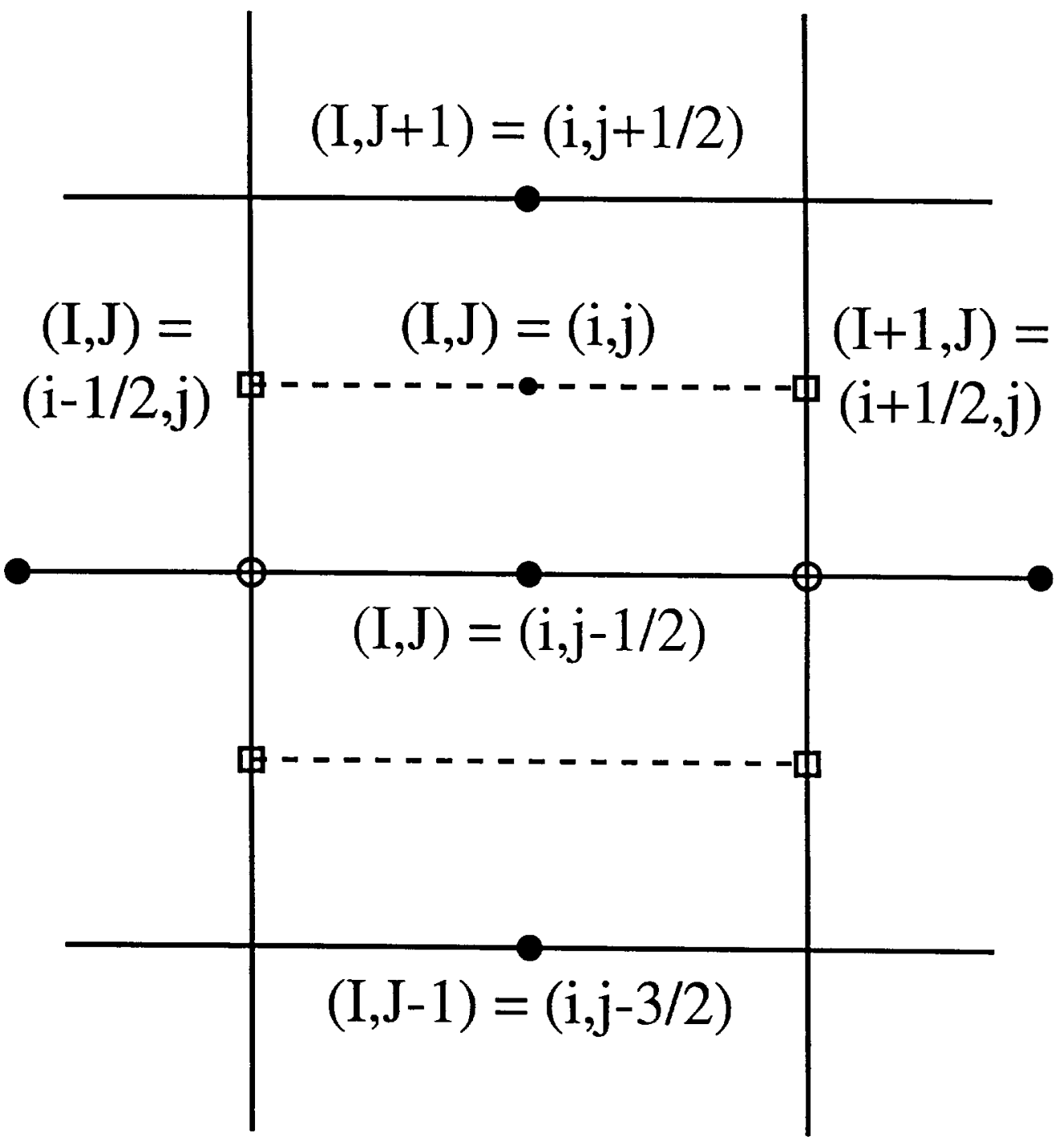

Figure 2: Stencil for the $z$ component of the momentum. Horizontal velocity $u_{x}=u$ is defined at the open squares $(i \pm 1 / 2, j)$, and vertical velocity $u_{z}=v$ is defined at the large filled circles at $(i, j \pm 1 / 2)$. All other quantities are defined at cell centers $(i, j)$. 


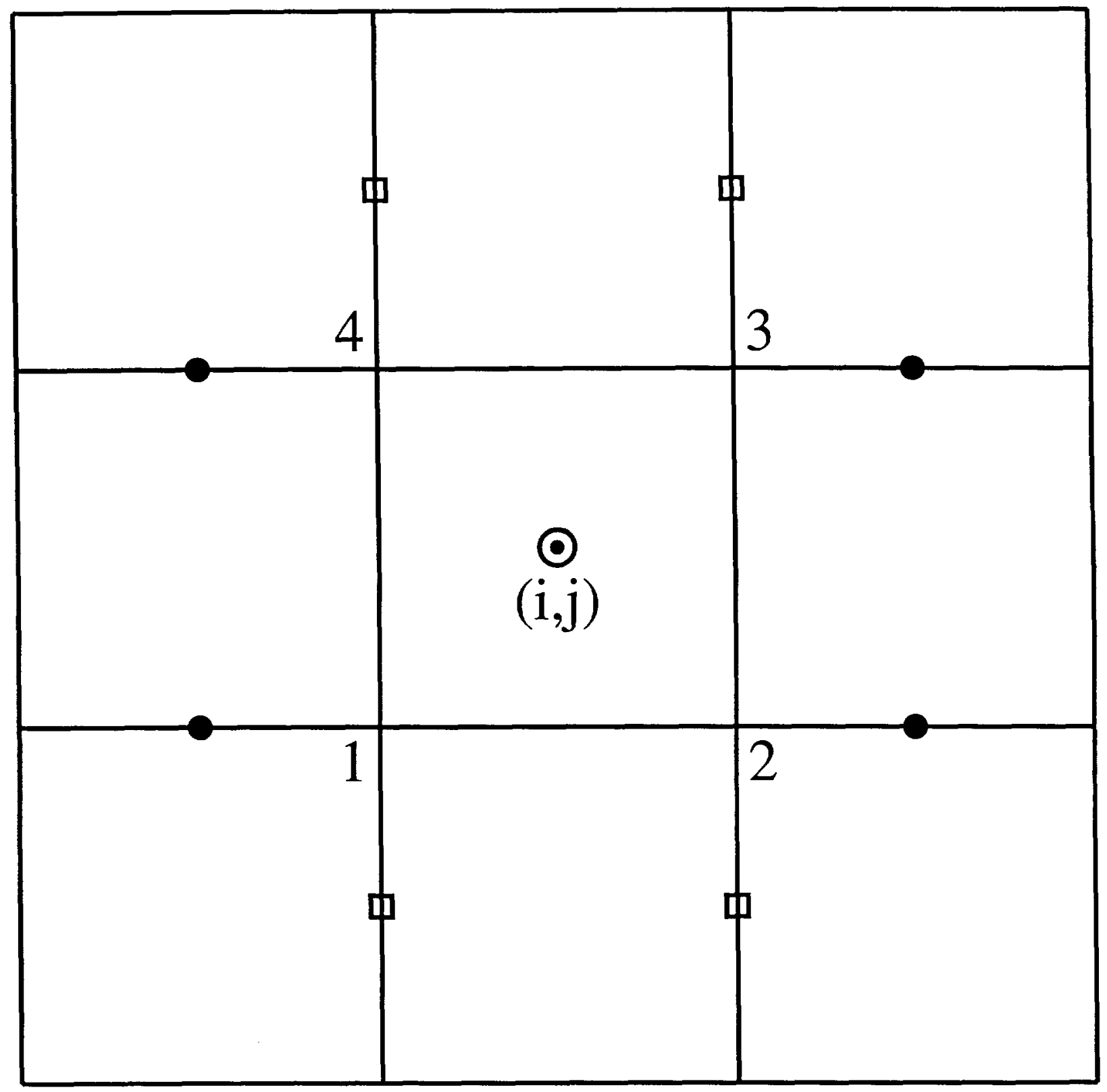

Figure 3: Stencil for the old differencing of the dissipation term, equation 46. The bullseye marks the cell center at which we evaluate the dissipation. The open squares mark the locations of the values of $u$ and the filled circles mark the locations of the values of $v$ used to evaluate the off-diagonal $\partial u / \partial z+\partial v / \partial x$ term. 


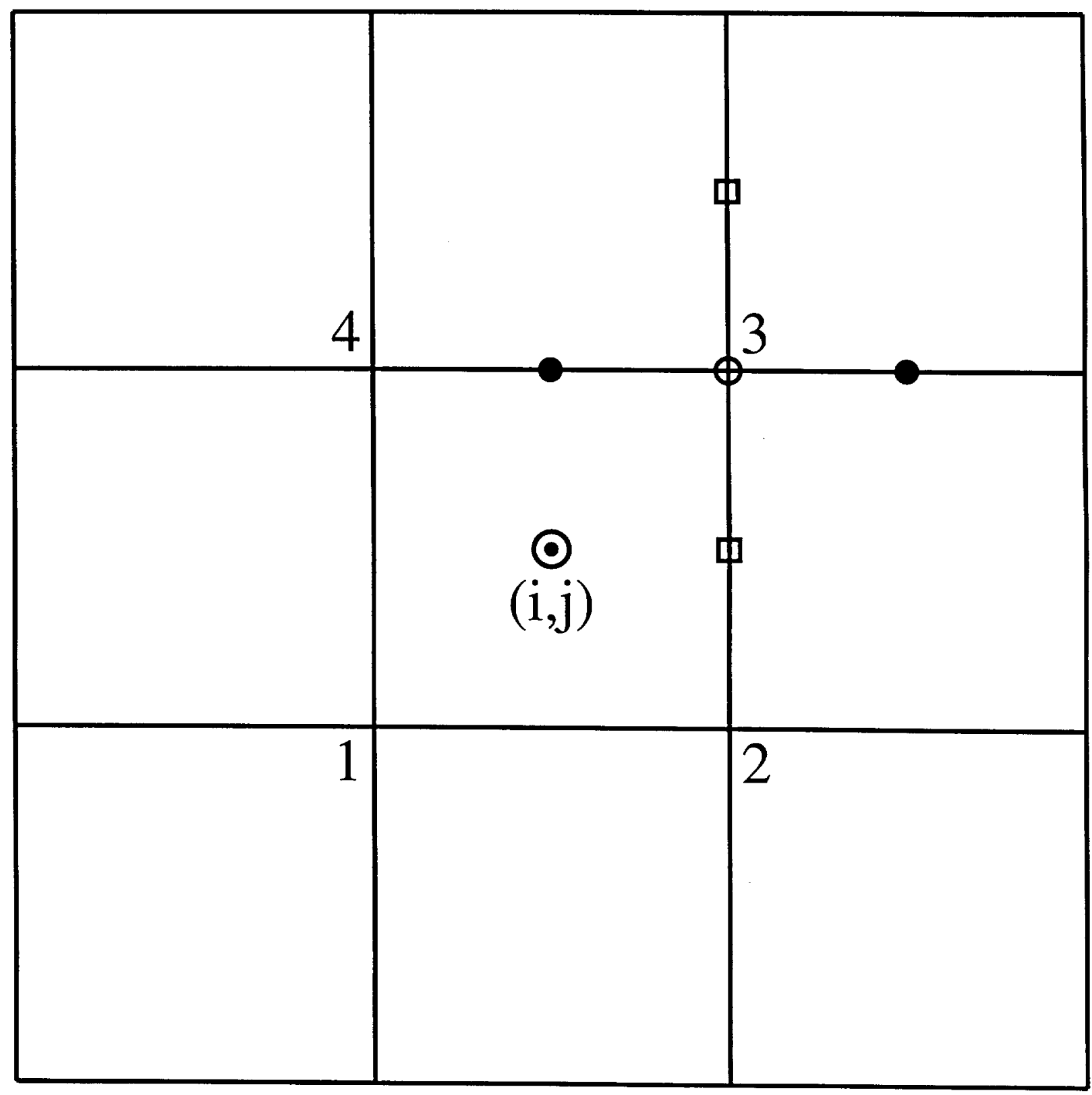

Figure 4: Stencil for the new differencing of the off-diagonal term in the rate of strain evaluated at corner 3, equation 47. Symbols are otherwise the same as in figure 3. 
Y 1 cycle $=0 \mathrm{t}=0.000000 \mathrm{D}+00 \mathrm{dt}=1.000000 \mathrm{D}-06$

$\max =8.000000 \mathrm{D}-01 \mathrm{~min}=2.000000 \mathrm{D}-01 \mathrm{dq}=2.000000 \mathrm{D}-01$

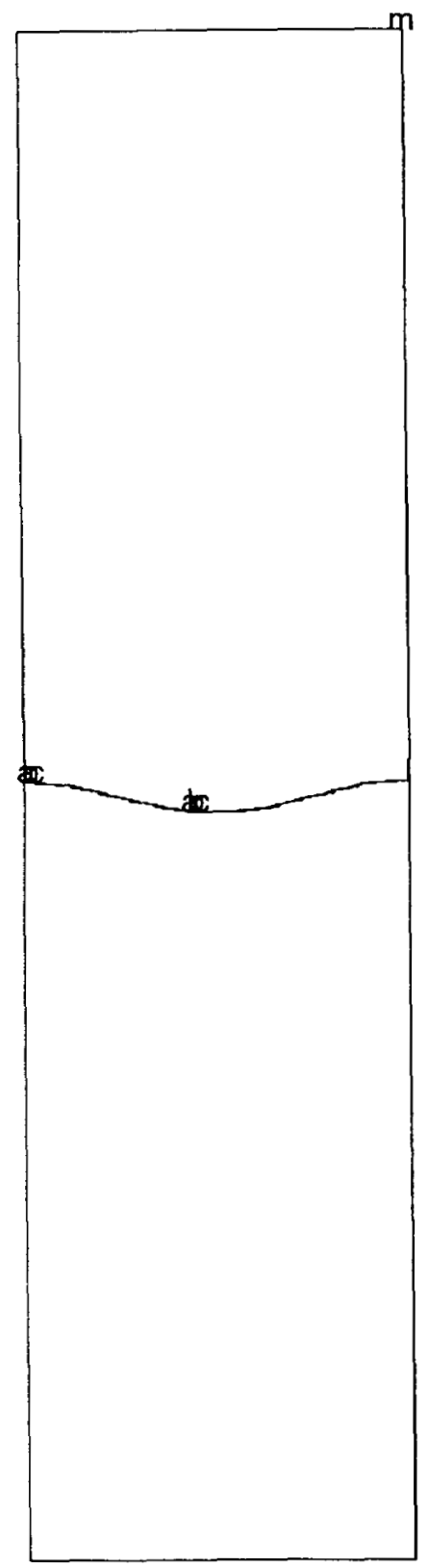

Figure 5: Mass fraction contours for species 1 (the heavy species) at $t=0$. In figures 5 through 10 , three contours are plotted with values of $0.2,0.5$, and 0.8 . These contours are labelled a through $c$, respectively. 
$Y 1$ cycle $=14556 \mathrm{t}=5.000192 \mathrm{D}-02 \mathrm{dt}=2.928830 \mathrm{D}-06$
$\max =8.000000 \mathrm{D}-01 \mathrm{~min}=2.000000 \mathrm{D}-01 \mathrm{dq}=2.000000 \mathrm{D}-01$

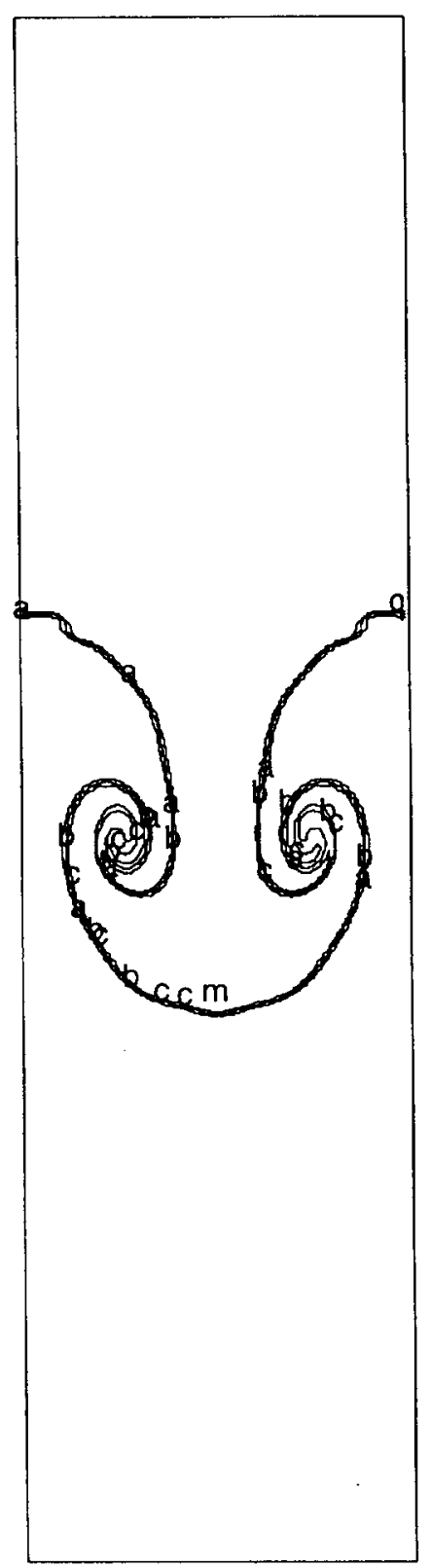

Figure 6: Mass fraction contours for species 1 for the old differencing method at $t=50 \mathrm{~ms}$. 
$Y \quad 1$ cycle $=14583 \mathrm{t}=5.000040 \mathrm{D}-02 \mathrm{dt}=2.998858 \mathrm{D}-06$
$\max =8.000000 \mathrm{D}-01 \mathrm{~min}=2.000000 \mathrm{D}-01 \mathrm{dq}=2.000000 \mathrm{D}-01$

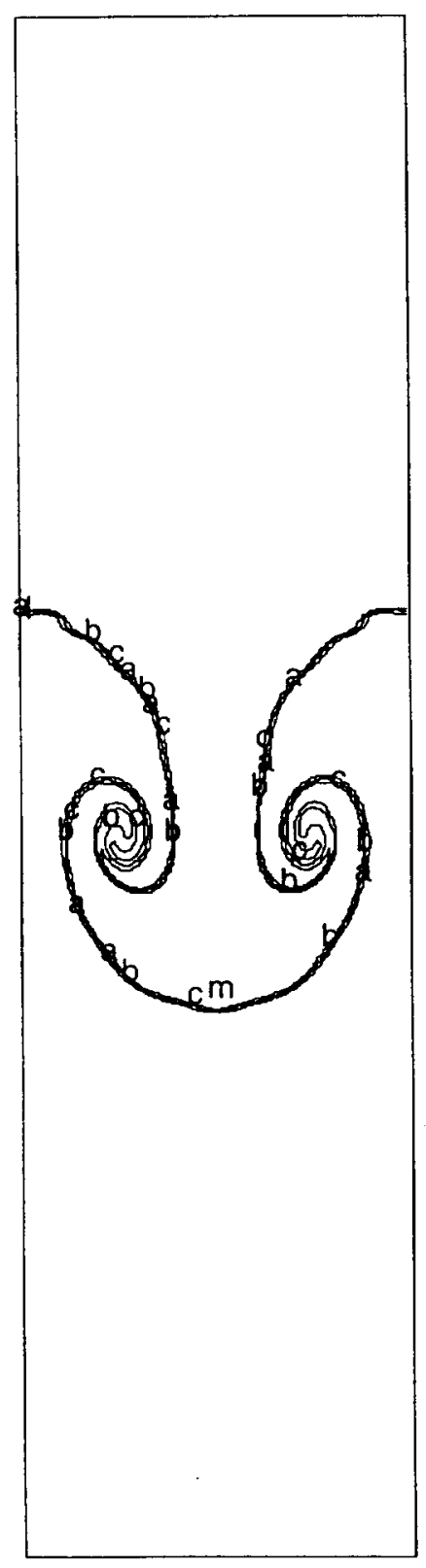

Figure 7: Mass fraction contours for species 1 for the new differencing method at $t=50 \mathrm{~ms}$. 
Y 1 cycle $=41695 \mathrm{t}=1.100001 \mathrm{D}-01 \mathrm{dt}=2.195600 \mathrm{D}-06$

$\max =1.100000 \mathrm{D}+00 \mathrm{~min}=2.000000 \mathrm{D}-01 \mathrm{dq}=3.000000 \mathrm{D}-01$

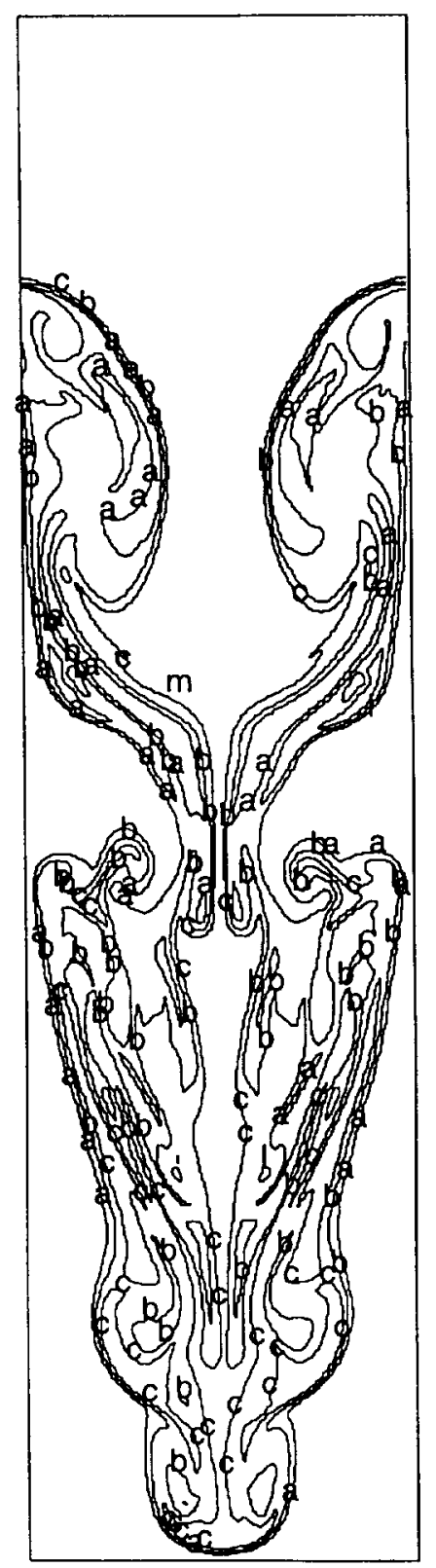

Figure 8: Mass fraction contours for species 1 for the old differencing method at $t=110 \mathrm{~ms}$. 
Y 1 cycle $=40976 \mathrm{t}=1.100017 \mathrm{D}-01 \mathrm{dt}=2.067614 \mathrm{D}-06$ $\max =1.100000 \mathrm{D}+00 \min =2.000000 \mathrm{D}-01 \mathrm{dq}=3.000000 \mathrm{D}-01$

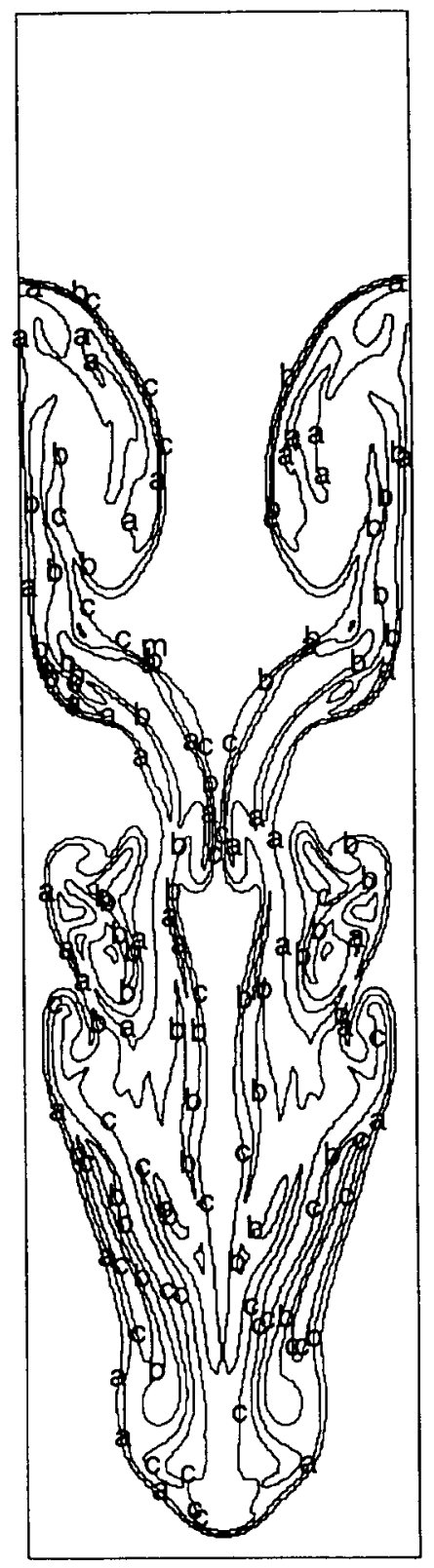

Figure 9: Mass fraction contours for species 1 for the new differencing method at $t=110 \mathrm{~ms}$. 
Y 1 cycle $=24101 \mathrm{t}=1.100025 \mathrm{D}-01 \mathrm{dt}=4.600000 \mathrm{D}-06$ $\max =8.000000 \mathrm{D}-01 \mathrm{~min}=2.000000 \mathrm{D}-01 \mathrm{dq}=2.000000 \mathrm{D}-01$

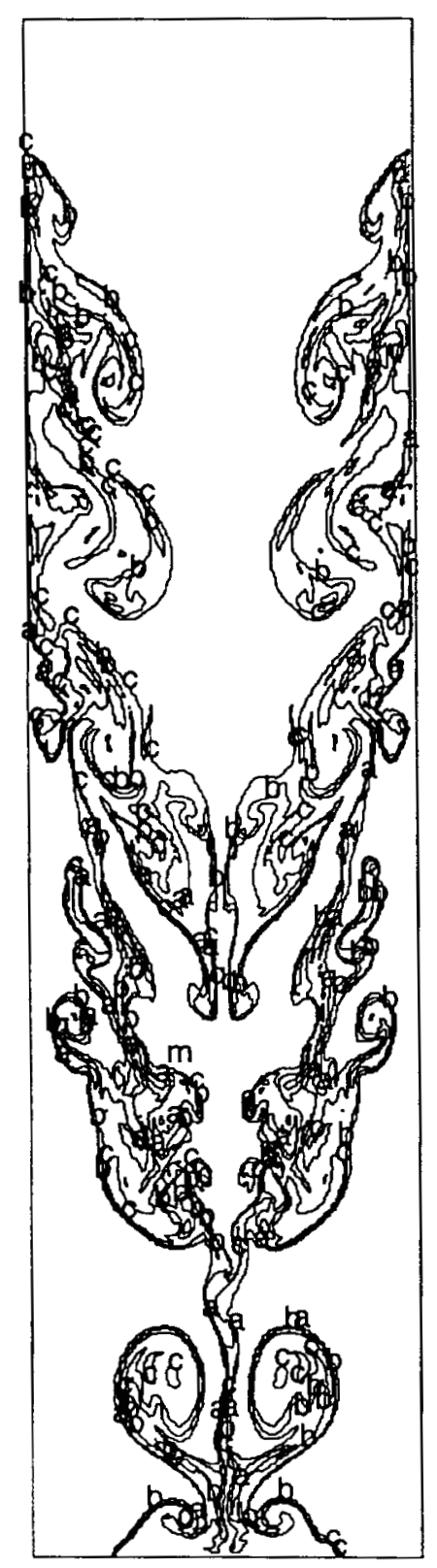

Figure 10: Mass fraction contours for species 1 for the new differencing method at $t=110 \mathrm{~ms}$ but with no turbulence model. 


\section{A COYOTE Input File}

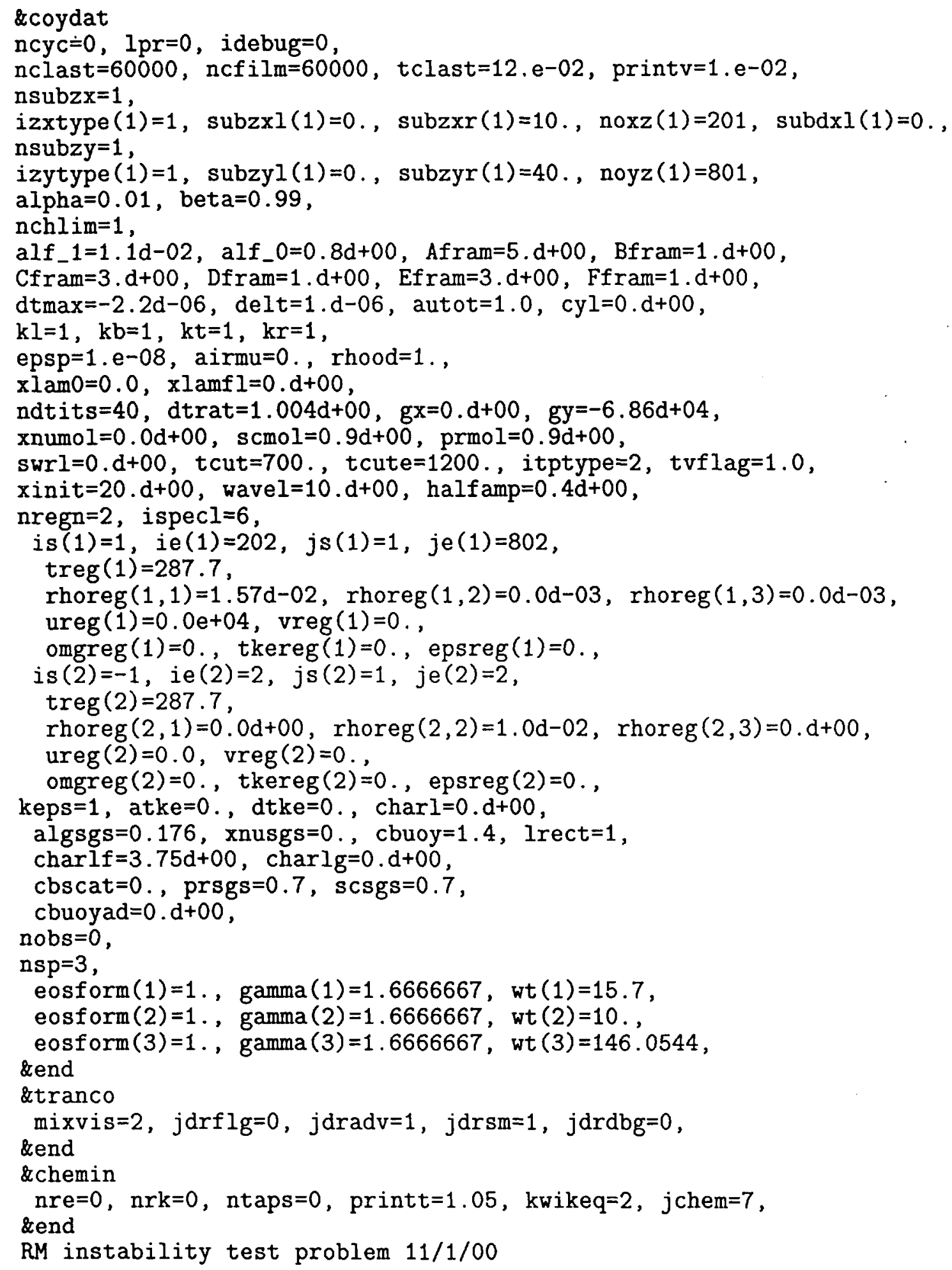

